\title{
TOXICIDADE DE PRODUTOS FORMULADOS À BASE DE FUNGOS ENTOMOPATOGÊNICOS PARA O CARUNCHO-DO-MILHO
}

\author{
MARCO AURÉLIO GUERRA PIMENTEL ${ }^{1}$ e EDICARLOS GOMES FERREIRA ${ }^{2}$
}

${ }^{1}$ Pesquisador,Embrapa Milho e Sorgo, Sete Lagoas, MG, Brasil, mpimentel@cnpms.embrapa.br

${ }^{2}$ Engenheiro Agrônomo,Unipam, Patos de Minas, MG,Brasil, edicarlos@unipam.edu.br

Revista Brasileira de Milho e Sorgo, v.11, n.2, p. 209-215, 2012

\begin{abstract}
RESUMO - Objetivou-se avaliar a toxicidade de produtos formulados à base dos fungos entomopatogênicos Beauveria bassiana (Balsamo) Vuillemin e Metarhizium anisopliae (Metschnikoff) Sorokin (Deuteromycotina: Hyphomycetes) sobre o caruncho-do-milho, Sitophilus zeamais Motschulsky (Coleoptera: Curculionidae). Adultos de S. zeamais foram expostos a grãos de milho (Zea mays L.) pulverizados com inseticidas formulados à base de B. bassiana $\left(\right.$ Boveril $\left.^{\circledR}\right)$ nas concentrações de $0,05 \mathrm{~g}$ a 2,0 g e $M$. anisopliae (Metarril ${ }^{\circledR}$ ) nas concentrações de $20 \mathrm{ml}$ a $250 \mathrm{ml}$ por litro de água destilada, por três, seis e 10 dias após a infestação. A mortalidade de $S$. zeamais aumentou com o incremento do período de exposição e com o aumento das concentrações dos inseticidas. O controle de $S$. zeamais pelos inseticidas à base de B. bassiana e $M$. anisopliae é uma alternativa aos inseticidas organossintéticos convencionais no manejo integrado de pragas de grãos armazenados.

Palavras-chave: Sitophilus zeamais, Beauveria bassiana, Metarhizium anisopliae, controle biológico, armazenamento de grãos.
\end{abstract}

\section{TOXICITY OF PRODUCTS FORMULATED WITH ENTOMOPATHOGENIC FUNGI ON THE MAIZE WEEVIL}

\begin{abstract}
The aim of this work was to test the toxicity of the entomopathogenic fungi Beauveria bassiana (Balsamo) Vuillemin e Metarhizium anisopliae (Metschnikoff) Sorokin (Deuteromycotina: Hyphomycetes) on the maize weevil Sitophilus zeamais Motschulsky (Coleoptera: Curculionidae). Adults of S. zeamais were exposed to maize (Zea mays L.) grains sprayed with insecticides based on B. bassiana (Boveril ${ }^{\circledR}$ ) in concentrations ranging from 0.05 to $2.0 \mathrm{~g}$ and $M$. anisopliae $\left(\right.$ Metarril $^{\circledR}$ ) at 20 to $250 \mathrm{ml}$ per liter on distilled water, for three, six and 10 days after infestation. The mortality of $S$. zeamais increased with the increment of the exposure period and with increasing concentrations of insecticides. The control of $S$. zeamais by insecticides based on B. bassiana and M. anisopliae is an alternative to conventional organo-synthetic insecticides in integrated pest management of stored grains.
\end{abstract}

Key words: Sitophilus zeamais, Beauveria bassiana, Metarhizium anisopliae, biological control, grain storage. 
Elevadas perdas de grãos em pós-colheita são registradas em decorrência de problemas logísticos, do ataque de insetos-praga e da ação de fungos. As perdas devido ao ataque de pragas no milho (Zea mays L.) durante o armazenamento chegam a $15 \%$ da produção de grãos (Santos, 2008), demandando pesquisas no manejo integrado de pragas (MIP). Entre os insetos-praga que causam danos aos grãos de milho, destaca-se o caruncho-do-milho Sitophilus zeamais Motschulsky (Coleoptera: Curculionidae) (Santos, 2008).

Dentre as principais medidas de controle do caruncho-do-milho tem-se o uso de inseticidas organossintéticos (piretróides e fosforados) e o expurgo dos grãos com fosfina (Santos, 2008; Pimentel et al., 2009). Apesar da eficiência de controle, o uso contínuo destes produtos por mais de três décadas tem resultado na resistência do caruncho-do-milho a inseticidas (Pereira et al., 2009; Pimentel et al., 2009). Assim, a busca por medidas alternativas faz-se necessária para o controle de pragas, oferecendo maior segurança, seletividade, biodegradabilidade, viabilidade econômica e aplicabilidade em programas de MIP com baixo impacto ambiental (Viegas Júnior, 2003; Michereff Filho et al., 2009).

O controle biológico com fungos entomopato-gênicos é uma alternativa ao controle químico (Batta, 2005; Cherry et al., 2005; Alves et al., 2008; Shams et al., 2011). A eficiência de fungos entomopatogênicos no controle de pragas de grãos armazenados, especialmente da ordem Coleoptera, tem sido investigada (Moino Júnior \& Alves, 1997; Batta, 2005; Cherry et al., 2005; Potrich et al., 2006; Pissinati et al., 2009; Shams et al., 2011), com ênfase na avaliação das espécies Beauveria bassiana (Balsamo) Vuillemin e Metarhizium anisopliae (Metschnikoff) Sorokin (Deuteromycotina: Hyphomycetes). O controle com fungos entomopatogênicos apresenta vantagens por não ser poluente, não provocar desequilíbrios biológicos e apresentar efeito residual, prolongando a proteção dos grãos armazenados. Além disso, não é tóxico para o homem e para os animais e pode ser aplicado com as máquinas convencionais, além de ser específico para insetos, não se desenvolvendo nos grãos durante o armazenamento (Alves et al., 2008; Michereff Filho et al., 2009).

Os fungos entomopatogênicos $B$. bassiana e M. anisopliae têm demonstrado eficiência no controle de pragas de produtos armazenados, como Callosobruchus maculatus (F.) (Coleoptera: Bruchidae) (Cherry et al., 2005; Shams et al., 2011), Rhyzopertha dominica (F.) (Coleoptera: Brostrichidae) (Batta, 2005), Tribolium castaneum (Herbst) (Coleoptera: Tenebrionidae), Sitophilus oryzae (L.) (Coleoptera: Curculionidae) (Padín et al., 2002), S. zeamais (Potrich et al., 2006), Sitophilus granarius (L.) (Coleoptera: Curculionidae) (Shams et al., 2011) e Caryedon serratus (Olivier) (Coleoptera: Bruchidae) (Ekesi et al., 2001). Contudo, grande parte dos trabalhos disponíveis na literatura avalia a eficiência de isolados através de pulverização sobre os insetos ou por imersão dos insetos em solução com os fungos. Ainda são escassos trabalhos que avaliam a eficácia de produtos comerciais formulados à base destes fungos entomopatogênicos em aplicação protetora diretamente nos grãos de milho, simulando as aplicações realizadas nas unidades de armazenamento de grãos e sementes.

Desta forma, objetivou-se avaliar a toxicidade de produtos formulados à base dos fungos entomopatogênicos B. bassiana e $M$. anisopliae sobre $S$. zeamais, além de estimar as concentrações e o tempo letal para os produtos comerciais por período de exposição. 
Os bioensaios foram realizados utilizando-se insetos adultos e não-sexados de $S$. zeamais que apresentavam entre sete e 10 dias de emergidos, obtidos de uma criação mantida em laboratório utilizando milho como substrato alimentar (13\% de umidade) e sem tratamento inseticida ou infestação.

A mortalidade de S. zeamais foi avaliada aos três, seis e 10 dias após a infestação, contando-se o número de mortos por concentração. $\mathrm{O}$ delineamento experimental foi inteiramente casualizado, em esquema fatorial, composto de nove concentrações, dois inseticidas $\left(\right.$ Boveril $^{\circledR}$ e Metarril $^{\circledR}$ ), três períodos de exposição e três repetições.

Os inseticidas avaliados foram o Boveril ${ }^{\circledR} \mathrm{WP}$ PL63 (i.a. B. bassiana 5\%) e o Metarril ${ }^{\circledR}$ WP E9 (i.a. M. anisopliae 5\%), que possuem garantia de 500 milhões de conídios viáveis por grama do produto. A calda foi preparada para cada inseticida e os grãos foram tratados via pulverização, utilizando-se atomizador manual. Para a pulverização, os grãos foram distribuídos uniformemente em uma bandeja plástica, de modo a se obter uma camada uniforme. Os grãos foram pulverizados a $20 \mathrm{~cm}$ de altura da massa de 1,0 $\mathrm{kg}$ de grãos, em local protegido do vento. $\mathrm{O}$ volume de calda aplicado foi de 2,0 $\mathrm{ml}$ por quilo de grãos, correspondendo a 2,0 1 de calda por $1000 \mathrm{~kg}$ de grãos. $\mathrm{O}$ atomizador foi calibrado em uma superfície de 1,0 $\mathrm{m}^{2}$ com $10 \mathrm{~kg}$ de grãos de milho, ajustando-se $2,0 \mathrm{ml}$ de solução por quilo de grãos de milho.

Após a pulverização, os grãos foram homogeneizados por agitação manual por dois minutos e, colocados em embalagens de papel com identificação dos tratamentos. Os bioensaios foram realizados em recipientes plásticos de $50 \mathrm{ml}(3,5 \mathrm{~cm}$ de diâmetro $\times$ $6,5 \mathrm{~cm}$ de altura) preenchidos com $60 \mathrm{~g}$ de grãos e infestados com 20 insetos adultos por recipiente. Os grãos dos tratamentos testemunha foram submetidos ao procedimento descrito anteriormente, aplicando somente água destilada.

A eficiência dos inseticidas foi determinada a partir de preparados das suspensões fúngicas nas concentrações de 0,$5 ; 0,75 ; 1,0 ; 1,5 ; 2,0 ; 2,5 ; 3,0 ; 4,0$; $5,0 \mathrm{~g}$ de Boveril ${ }^{\circledR}$ por litro de água destilada e 20; 50; $100 ; 125 ; 150 ; 175 ; 200 ; 225 ; 250 \mathrm{ml}$ de Metarril $^{\circledR}$ por litro de água destilada. Foi estipulado um intervalo de concentrações que contemplassem valores de mortalidade entre zero e $100 \%$.

Os dados de mortalidade foram corrigidos pela mortalidade apresentada na testemunha e, em seguida, foram submetidos à análise de Probit (SAS Institute, 2002), gerando as curvas de concentração-mortalidade para cada período de exposição e inseticidas e as curvas de tempo-mortalidade para as concentrações de $150 \mathrm{ml} \mathrm{l}^{-1}$ e $200 \mathrm{ml} \mathrm{l}^{-1}$ para Metarril ${ }^{\circledR}$ e 0,$5 ; 1,0$ e 2,0 g l$^{-1}$ para Boveril ${ }^{\circledR}$. A partir das curvas de concentração-mortalidade e tempo-mortalidade, foram estimadas as concentrações letais e os tempos letais para 50 e $95 \%$ da população de $S$. zeamais.

A mortalidade de $S$. zeamais aumentou com o período de exposição e na interação deste com as respectivas concentrações de Boveril ${ }^{\circledR}\left(\mathrm{F}_{26,80}=7,38\right.$; $\mathrm{p}<$ 0,0001) e para Metarril ${ }^{\circledR}\left(\mathrm{F}_{26,80}=20,39 ; \mathrm{p}<0,0001\right)$. As concentrações letais para $50 \%$ da população $\left(\mathrm{CL}_{50}\right)$ foram de 181,$9 ; 157,7$ e $83,5 \mathrm{ml} \mathrm{l}^{-1}$ para Metarril ${ }^{\circledR}$ e 2,1; 1,6 e 1,2 $\mathrm{g} \mathrm{l}^{-1}$ para Boveril ${ }^{\circledR}$, respectivamente (Tabela 1). As concentrações letais para $95 \%$ da população $\left(\mathrm{CL}_{95}\right)$ para Metarril ${ }^{\circledR}$ foram de 437,1; 388,7 e $554,2 \mathrm{ml} \mathrm{l}^{-1}$ e para Boveril ${ }^{\circledR}$ foram de 9,5; 7,4 e 7,2 $\mathrm{g}$ $1^{-1}$, respectivamente (Tabela 1 ). ${\mathrm{A} \mathrm{CL}_{50}}$ reduziu com o aumento do período de exposição aos inseticidas. Apesar da interposição dos intervalos de confiança das concentrações letais para três e seis dias de exposição de Metarril $^{\circledR}$, pode-se observar diferença dos valores estimados e tendência de redução dos valores 
de $\mathrm{CL}_{50}$ e $\mathrm{CL}_{95}$ com o aumento do período de exposição (Tabela 1).

A mortalidade de S. zeamais, tanto com Metarril $^{\circledR}$ quanto com Boveril ${ }^{\circledR}$, aumentou com o período de exposição, independente da concentração. O maior número de insetos infectados foi observado aos dez dias da aplicação. Isto se deve ao tempo demandado (72 h) pelos fungos entomopatogênicos para o crescimento, a colonização, a expressão dos sintomas, a morte e a esporulação (04-12 dias), ocasionando infecção em outros insetos (Batta, 2005). Os resultados obtidos corroboram com Moino Junior \& Alves (1997), os quais observaram, que, ao aumentar a concentração de $B$. bassiana, maior a mortalidade de $S$. zeamais para o controle de $S$. oryzae, $S$. zeamais e $R$. dominica. ao constatarem a ocorrência natural de $B$. bassiana em cascudinho-do-feijoeiro Aracanthus sp. (Coleoptera: Curculionidae), relataram que a mortalidade dos adultos aumentou com a exposição ao fungo.

As concentrações de Metarril $^{\circledR}$ que ocasionaram maior mortalidade foram $125 \mathrm{ml} \mathrm{l}^{-1}$ e $200 \mathrm{ml} \mathrm{l}^{-1}$, provocando 85 e $83 \%$ de mortalidade, respectivamente, aos dez dias após a aplicação. Para o Boveril ${ }^{\circledR}$, as concentrações que apresentaram maior mortalidade foram 4,0 $\mathrm{g} \mathrm{l}^{-1}$ e 5,0 $\mathrm{g} \mathrm{l}^{-1}$, com mortalidade média de 85,0 e $98,3 \%$, respectivamente, aos dez dias após a aplicação.

Os tempos letais estimados para $50 \%$ da população $\left(\mathrm{TL}_{50}\right)$ nas concentrações de $150 \mathrm{ml} \mathrm{l}^{-1}$ e 200 $\mathrm{ml} \mathrm{l}^{-1}$ de Metarril $^{\circledR}$ foram de 5,3 e 3,7 dias, respectivamente. As $\mathrm{TL}_{50}$ para as concentrações de 0,$5 ; 1,0$

TABELA 1. Concentrações letais de Metarril® e Boveril ${ }^{\circledR}$ para S. zeamais expostos a grãos de milho tratados por período de exposição.

\begin{tabular}{|c|c|c|c|c|c|}
\hline $\begin{array}{c}\text { Inseticida / } \\
\text { Período de Exposição } \\
\text { (dias após a aplicação) }\end{array}$ & $\mathrm{N}^{1}$ & $\mathrm{CL}^{250}\left(\mathrm{IC} 95 \%{ }^{2}\right)$ & CL95 (IC 95\%33) & $\chi^{2}$ & $\mathrm{p}^{*}$ \\
\hline Metarril@ & \multicolumn{3}{|c|}{$\left(\mathrm{ml} \mathrm{l}^{-1}\right)$} & & \\
\hline 3 & 540 & $181,9(167,7-201,6)$ & $437,1(350,3-630,7)$ & 7,82 & 0,17 \\
\hline 6 & 540 & $157,7(144,8-171,1)$ & $388,7(329,0-497,0)$ & 8,17 & 0,15 \\
\hline 10 & 540 & $83,5(69,0-100,0)$ & $554,2(383,4-971,4)$ & 3,97 & 0,41 \\
\hline Boveril $\AA$ & \multicolumn{4}{|c|}{$\left(\mathrm{g} \mathrm{l}^{-1}\right)$} & \\
\hline 3 & 540 & $2,1(1,8-2,3)$ & $9,5(7,4-13,3)$ & 8,64 & 0,28 \\
\hline 6 & 540 & $1,6(1,4-1,8)$ & $7,4(5,9-10,1)$ & 10,82 & 0,15 \\
\hline 10 & 540 & $1,2(1,0-1,4)$ & $7,2(5,4-11,1)$ & 10,15 & 0,12 \\
\hline
\end{tabular}

${ }^{1} N=$ Número de insetos por bioensaio. ${ }^{2} \mathrm{CL}=$ Concentração letal. ${ }^{3} \mathrm{IC} 95 \%=$ Intervalo de confiança a 95\% de probabilidade. $\chi^{2}=$ Quiquadrado. $\mathrm{p}=$ Probabilidade $\left({ }^{*} \mathrm{p}>0,05\right)$

O tratamento preventivo dos grãos com fungos pode retardar o desenvolvimento de $R$. dominica em oito a 12 dias (Batta, 2005). Assim, o controle de $R$. dominica com $M$. anisopliae pode ser promissor no MIP de produtos armazenados. Pissinati et al. (2009),

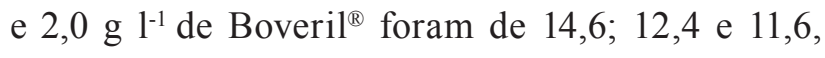
respectivamente (Tabela 2). Os tempos letais para 95\% da população $\left(\mathrm{TL}_{95}\right)$ nas concentrações de 150 $\mathrm{ml} \mathrm{l}^{-1}$ e $200 \mathrm{ml} \mathrm{l}^{-1}$ de Metarril ${ }^{\circledR}$ foram de 26,8 e 21,9 dias, respectivamente, enquanto para as concentra- 
ções de 0,$5 ; 1,0$ e 2,0 $\mathrm{g} \mathrm{l}^{-1}$ de Boveril ${ }^{\circledR}$ foram estimadas em mais de 129 dias (Tabela 2). Observou-se redução do $\mathrm{TL}_{50}$ com o aumento da concentração dos inseticidas, sem interposição dos intervalos de confiança nas concentrações avaliadas. Os valores estimados de $\mathrm{TL}_{95}$ para o inseticida Boveril ${ }^{\circledR}$ foram superiores a 129 dias na concentração de 2,0 $\mathrm{g} \mathrm{l}^{-1}$, tempo elevado em relação à $\mathrm{TL}_{95}$ para o inseticida Metarril $^{\circledR}$. zenadoras, processadoras de milho e indústrias de sementes, visando ao controle preventivo (Cherry et al., 2005; Santos, 2008).

A aplicação de produtos formulados à base de fungos entomopatogênicos em grãos de milho pode ser uma alternativa ao controle químico no MIP de grãos armazenados, pois pode ocorrer nas condições atuais das estruturas armazenadoras, sem grandes modificações estruturais. As condições climáticas do

TABELA 2. Tempos letais de Metarril ${ }^{\circledR}$ e Boveril ${ }^{\circ}$ para $S$. zeamais expostos a grãos de milho tratados por concentração

\begin{tabular}{|c|c|c|c|c|c|}
\hline $\begin{array}{l}\text { Inseticida/ } \\
\text { Concentração }\end{array}$ & $\mathrm{N}^{1}$ & $\mathrm{TL}^{2}{ }_{50}\left(\mathrm{IC} 95 \%{ }^{3}\right)$ & $\mathrm{TL}_{95}$ (IC 95\%) & $\chi^{2}$ & $p^{*}$ \\
\hline Metarril@ & \multicolumn{4}{|c|}{ (dias) } & \\
\hline $150 \mathrm{ml} \mathrm{l}^{-1}$ & 180 & $5,3(4,3-6,5)$ & $26,8(16,8-74,9)$ & 0,92 & 0,34 \\
\hline $200 \mathrm{ml} \mathrm{l}^{-1}$ & 180 & $3,7(2,5-4,6)$ & $21,9(13,8-66,9)$ & 0,23 & 0,63 \\
\hline Boveril $\AA$ & \multicolumn{4}{|c|}{ (dias) } & \\
\hline $0,5 \mathrm{~g} \mathrm{l}^{-1}$ & 180 & $14,6(9,2-135,9)$ & $>255,2$ & 0,05 & 0,83 \\
\hline $1,0 \mathrm{~g} \mathrm{l}^{-1}$ & 180 & $12,4(8,6-43,0)$ & $>147,6$ & 0,13 & 0,71 \\
\hline $2,0 \mathrm{~g} \mathrm{l}^{-1}$ & 180 & $11,6(8,2-33,6)$ & $>129,9$ & 0,01 & 0,92 \\
\hline
\end{tabular}

${ }^{1} N=$ Número de insetos por bioensaio. ${ }^{2} \mathrm{TL}=$ Tempo letal. ${ }^{3} \mathrm{IC} 95 \%$ = Intervalo de confiança a $95 \%$ de probabilidade. $\chi^{2}=$ Qui-quadrado. $\mathrm{p}=$ Probabilidade $\left({ }^{*} \mathrm{p}>0,05\right)$

A determinação de concentrações de fungos entomopatogênicos para o controle de pragas de produtos armazenados tem como metodologia a pulverização de suspensões aquosas dos propágulos do patógeno sobre os insetos ou até mesmo a imersão dos insetos nas soluções (Moino Junior \& Alves, 1997; Lord, 2007; Potrich et al., 2006; Alves et al., 2008; Shams et al., 2011). Isto dificulta a comparação dos resultados obtidos, devido à diferença entre metodologias. Entretanto, buscou-se aproximação com a aplicação comercial dos microrganismos no ambiente de armazenamento com a pulverização direta sobre os grãos de milho, o que é feito nas unidades arma- ambiente de armazenamento não constituem impedimento à aplicação de inseticidas à base de fungos, pois baixa umidade do ambiente de armazenamento de grãos não impedem o uso de $B$. bassiana no MIP de grãos armazenados (Lord, 2007).

\section{Conclusões}

Os produtos formulados à base dos fungos entomopatogêncios $B$. bassiana e $M$. anisopliae apresentam toxicidade ao caruncho-do-milho S. zeamais, constituindo alternativa ao controle químico no MIP de produtos armazenados. 


\section{Referências}

ALVES, S. B.; LOPES, R. B.; VIEIRA, S. A.; TAMAI, M. A. Fungos entomopatogênicos usados no controle de pragas na América Latina. In: ALVES, S. B.; LOPES, R. B. (Ed.). Controle microbiano de pragas na América Latina. Piracicaba: FEALQ, 2008. p. 69-110.

BATTA, Y.A. Control of the lesser grain borer (Rhyzopertha dominica (F.), Coleoptera: Bostrichidae) by treatments with residual formulations of Metarhizium anisopliae (Metschnikoff) Sorokin (Deuteromycotina: Hyphomycetes). Journal of Stored Products Research, Oxford, v. 41, n. 2, p. 221-229, 2005.

CHERRY, A. J.; ABALOB, P.; HELL, K. A laboratory assessment of the potential of different strains of the entomopathogenic fungi Beauveria bassiana (Balsamo) Vuillemin and Metarhizium anisopliae (Metschnikoff) to control Callosobruchus maculatus (F.) (Coleoptera: Bruchidae) in stored cowpea. Journal of Stored Products Research, Oxford, v. 41,n. 3, p. 295-309, 2005. EKESI, S.; EGWURUBE, E. A.; AKPA, A. D.; ONU, I. Laboratory evaluation of the entomopathogenic fungus, Metarhizium anisopliae for the control of the groundnut bruchid, Caryedon serratus on groundnut. Journal of Stored Products Research, Oxford, v. 37, n. 4, p. 313-321, 2001.

LORD, J. C. Desiccation increases the efficacy of Beauveria bassiana for stored-grain pest insect control. Journal of Stored Products Research, Oxford, v. 43, n. 4, p. 535-539, 2007.

MICHEREFF FILHO, M.; FARIA, M.; WRAIGHT, S. P.; SILVA, K. F. A. S. Micoinseticidas e micoacaricidas no Brasil: como estamos após quatro décadas? Arquivos do Instituto Biológico, São Paulo, v. 76, n. 4, p. 769-779, 2009.

MOINO JUNIOR, A.; ALVES, S. B. Determinação de concentrações de Beauveria bassiana (Bals.)
Vuill. para o controle de insetos-pragas de grãos armazenados. Anais da Sociedade Entomológica do Brasil, Jaboticabal, v. 26, n. 1, p. 15-19, 1997.

PADÍN, S.; DAL BELLO, G.; FABRIZIO, M. Grain loss caused by Tribolium castaneum, Sitophilus oryzae and Acanthoscelides obtectus in stored durum wheat and beans treated with Beauveria bassiana. Journal of Stored Products Research, Oxford, v. 38, n. 1, p. 69-74, 2002.

PEREIRA, C. J.; PEREIRA, E. J. G.; CORDEIRO, E. M. G.; DELLALUCIA, T. M. C.; TÓTOLA, M. R.; GUEDES, R. N. C. Organophosphate resistance in the maize weevil Sitophilus zeamais: Magnitude and behavior. Crop Protection, Guildford,v.28,n.2,p.168-173,2009. PIMENTEL, M. A. G.; FARONI, L. R. D’A.; GUEDES, R. N. C.; SOUSA, A. H.; TÓTOLA, M. R. Phosphine resistance in Brazilian populations of Sitophilus zeamais Motschulsky (Coleoptera: Curculionidae). Journal of Stored Products Research, Oxford, v. 45, n. 1, p. 7174, 2009.

PISSINATI, A.; MIKAMI,A. Y; FAGOTTI, D; VENTURA, M. U. Ocorrência do fungo entomopatogênico Beauveria bassiana (Balsamo) Vuill. em população de Aracanthus sp. (Coleoptera: Curculionidae) em feijoeiro. Biotemas, Florianópolis, v. 22, n. 4, p. 219223, 2009.

POTRICH, M.; ALVES, L. F. A.; MERTZ, N. R.; SILVA, E. R. L. Avaliação de Beauveria bassiana (Bals.) Vuill. e Metarhizium anisopliae (Metsch.) Sorok. para controle de Sitophilus zeamais (Coleoptera: Curculionidae). BioAssay, Londrina, v. 1, n. 12, p. $1-9,2006$.

SANTOS, J. P. Controle de pragas durante o armazenamento de milho. In: CRUZ, J. C.; KARAM, D.; MONTEIRO, M. A. R.; MAGALHÃES, P. C. (Ed.). A culturado milho. Sete Lagoas: Embrapa Milho e Sorgo, 2008. p. 257-302. 
SAS INSTITUTE. SAS/STAT User's Guide. Version 8.02. Cary, 2002.

SHAMS, G.; SAFARALIZADEH, M. H.; IMANI, S.; SHOJAI, M.; ARAMIDEH, S. A laboratory assessment of the potential of the entomopathogenic fungi Beauveria bassiana (Beauvarin ${ }^{\circledR}$ to control Callosobruchus maculatus (F.) (Coleoptera: Bruchidae) and Sitophilus granarius (L.) (Coleoptera: Curculionidae). African Journal of Microbiology Research, Nairobi, v.5,n.10,p. 1192-1196, 2011.

VIEGAS JÚNIOR, J. C. Terpenos com atividade inseticida: uma alternativa para o controle químico de insetos. Química Nova, São Paulo, v. 26, n. 3, p. 390-400, 2003. 